\title{
ESTUDO MECÂNICO DO CONCRETO DE ALTO DESEMPENHO COM AGREGADO RECICLADO
}

\section{MECHANICAL BEHAVIOR OF HIGH PERFORMANCE CONCRETE WITH RECYCLED AGGREGATES}

\section{Maria da Consolação Fonseca Albuquerque ${ }^{1}$, Aparecido Carlos Gonçalves ${ }^{2}$}

${ }^{1}$ Professor Doutor, Departamento de Engenharia, Universidade Estadual Paulista -

UNESP - Campus de Ilha Solteira e-mail: sao@dec.feis.unesp.br

Alameda Bahia, 550 - Centro - 15385-000 - Ilha Solteira - SP - Fone: (18) 3743-

1213.

${ }^{2}$ Professor Adjunto, Departamento de Engenharia, Universidade Estadual Paulista -

UNESP - Campus de Ilha Solteira e-mail: cido@dem.feis.unesp.br

Avenida Brasil, 56 - Centro - 15385-000 - Ilha Solteira - SP - Fone: (18) 3743-1038.

\section{RESUMO}

A utilização de agregados reciclados em concreto, além de minimizar o impacto ambiental diminui o enorme consumo de fontes naturais. Entretanto, os agregados reciclados não são usados na produção de Concreto de Alto Desempenho (CAD) devido à alta capacidade de absorção de água, propriedades instáveis e fraca resistência. Tais dificuldades podem ser minimizadas com a seleção e a substituição parcial do agregado. No presente trabalho os agregados, gerados de demolição das construções, foram selecionados e diferentes porcentagens do mesmo foram usadas em substituição ao agregado natural para confecção do CAD. Os resultados mostraram que o agregado reciclado pode substituir parcialmente o agregado natural, para o mesmo traço, sem perda de resistência e com uma alteração bastante pequena na relação água/ cimento das composições estudadas, havendo necessidade de uma maior quantidade de água para se obter um abatimento igual ao obtido para o concreto sem adição de resíduo.

Palavras-chave: Concreto de alto desempenho. Resíduo. Propriedades mecânicas

ABSTRACT

The utilization of recycled aggregates can minimize environmental impact, and decreases the consumption of natural resources used for concrete applications. 
However, recycled aggregates are not suitable for use in the production of High Performance Concrete (HPC) due to their relatively high absorption capacity, instable properties and weaker strength. Such difficulties can be overcome through selection and partial substitution of aggregates. In this paper, recycled aggregates generated from demolished-construction wastes were selected and different perceptual were substituted through natural aggregates for application in the HPC. Results show that specimens containing partially recycled aggregates are optimum for producing normal strength recycled aggregates concrete.

Keywords: High-performance concrete. Residue. Mechanical properties

\section{INTRODUÇÃO}

O reaproveitamento de resíduos aparece nos dias atuais como questão fundamental na melhoria do meio ambiente, tanto em função da redução de desperdícios de recursos naturais, quanto na minimização dos impactos ambientais oriundos da disposição final dos resíduos.

A fabricação de concreto não tem sido ecologicamente correta, devido a sua natureza de consumo destrutivo dos recursos naturais e dos diversos impactos ambientais após seu uso. Levando em consideração o conceito de sustentabilidade, a indústria do concreto tem implementado diversas estratégias com o uso do concreto, como o melhoramento da durabilidade do concreto e melhor uso dos materiais reciclados.

Em geral os agregados ocupam de 55 a $80 \%$ do volume do concreto. Sem a alternativa de agregados reciclados, a indústria global de concreto consumiria de 8 a 12 bilhões de toneladas de agregados naturais anualmente após o ano 2010. Tal consumo causará a destruição do ambiente (TU et al., 2006).

Portanto, o uso de materiais reciclados tem se tornado cada vez mais popular. Geralmente os materiais reciclados são usados em concreto com a finalidade de substituir agregados miúdos, graúdos ou atuar como aditivo ao cimento. Muitos materiais de refugo são utilizados na manufatura de concreto normal e até mesmo de Concreto de Alto Desempenho (CAD) (AJDUKIEWICZ e KLISZCZEWICZ, 2002; POON et al., 2002; ÁTIS., 2005; BRITO et al., 2005; HUANG et al., 2005; TU et al., 2006).

Raramente os agregados reciclados, entretanto, são utilizados em construções estruturais devido à deficiência do material como a grande capacidade de absorção de água e sua forma angular e alongada (CHANDRA, 1997). A utilização de agregados reciclados no CAD, no entanto, é necessária, pois está sendo cada vez mais utilizado. Além de que, a utilização de agregados reciclados pode levar a indústria de concreto a abranger o conceito de desenvolvimento sustentável. Desse modo este estudo examina as propriedades mecânicas do CAD com o uso de agregados reciclados originados de concreto de demolição de construção, substituindo parcialmente e 
totalmente os agregados naturais miúdos e comparando os resultados com o concreto fabricado com agregados naturais.

\section{MATERIAL E MÉTODOS}

\subsection{Materiais}

O cimento utilizado neste estudo foi o Cimento Portland V - ARI - RS. A densidade do cimento foi de $3,12 \mathrm{~g} / \mathrm{cm}^{3}$ e sua análise química é mostrada na Tabela 1 . $\mathrm{O}$ aditivo mineral utilizado foi sílica ativa em pó, não densificada, cuja composição química é apresentada na Tabela 2 e o aditivo superplastificante utilizado foi o viscocrete $20 \mathrm{HE}$. $\mathrm{O}$ agregado graúdo foi basalto com tamanho nominal 4,8 $\mathrm{mm}$ (brita 00 - pedrisco) com módulo de finura de 4,94 e com tamanho nominal $19 \mathrm{~mm}$ (brita $01)$.

Foi usado agregado fino natural e reciclado. O natural foi areia média com módulo de finura de 2,27 e massa específica de $2,63 \mathrm{~g} / \mathrm{cm}^{3}$. O reciclado foi resíduo de concreto obtido das empresas coletoras de entulho da cidade de Ilha Solteira. Dos resíduos de construção das caçambas foi selecionado somente o concreto e posteriormente o mesmo passou por processo de britagem e peneiramento. O módulo de finura do resíduo foi de 2,60 e a massa específica de $2,63 \mathrm{~g} / \mathrm{cm}^{3}$.

Tabela 1 - Análise química do cimento

\begin{tabular}{|c|c|c|c|}
\hline \multirow[t]{2}{*}{ Elemento } & \multirow[t]{2}{*}{$(\%)$} & \multicolumn{2}{|c|}{$\begin{array}{c}\text { Especificações } \\
\text { NBR 5733:1991 } \\
\text { (ABNT, 1991) }\end{array}$} \\
\hline & & $\min$ & máx \\
\hline Perda ao fogo & 3,42 & - & 4,5 \\
\hline Insolúveis & 0,84 & - & 1,0 \\
\hline $\mathrm{SiO}_{2}$ & 20,88 & - & - \\
\hline $\mathrm{Fe}_{2} \mathrm{O}_{3}$ & 2,29 & - & - \\
\hline $\mathrm{Al}_{2} \mathrm{O}_{3}$ & 7,34 & - & - \\
\hline $\mathrm{CaO}$ & 60,43 & - & - \\
\hline $\mathrm{MgO}$ & 2,02 & - & 6,5 \\
\hline $\mathrm{SO}_{3}$ & 2,59 & - & 3,5 \\
\hline $\mathrm{Na}_{2} \mathrm{O}$ & 0,16 & - & - \\
\hline $\mathrm{K}_{2} \mathrm{O}$ & 0,77 & - & - \\
\hline Equiv alcalino $\mathrm{Na}_{2} \mathrm{O}$ & 0,61 & - & - \\
\hline Cal livre em $\mathrm{CaO}$ & 1,17 & - & - \\
\hline
\end{tabular}

Fonte: Scoaris, (2004) 
Tabela 2 - Composição química da sílica ativa

\begin{tabular}{|c|c|}
\hline Composição química & Quantidades $\mathbf{( \% )}$ \\
\hline Óxido de silício & $\left(\mathrm{SiO}_{2}\right) 91,00$ \\
\hline Óxido de alumínio & $\left(\mathrm{Al}_{2} \mathrm{O}_{3}\right) 0,10$ \\
\hline Óxido de ferro & $\left(\mathrm{Fe}_{2} \mathrm{O}_{3}\right) 0,70$ \\
\hline Óxido de cálcio & $(\mathrm{CaO}) 1,10$ \\
\hline Óxido de magnésio & $(\mathrm{MgO}) 1,50$ \\
\hline Óxido de sódio & $\left(\mathrm{Na}_{2} \mathrm{O}\right) 0,39$ \\
\hline Óxido de potássio & $\left(\mathrm{K}_{2} \mathrm{O}\right) 0,44$ \\
\hline Óxido de fósforo & $\left(\mathrm{P}_{2} \mathrm{O}_{5}\right) 0,10$ \\
\hline C (total) & 0,50 \\
\hline Perda ao fogo & 1,50 \\
\hline Total & 97,33 \\
\hline
\end{tabular}

Fonte: Silmix da Camargo Corrêa Cimentos SA

\subsection{Preparo das Misturas de Concreto}

Apresentam-se na Tabela 3 o traço que foi objeto de estudo e as porcentagens de agregado miúdo e de resíduo, em substituição ao mesmo, para cada mistura de CAD. Todas as misturas tiveram o acréscimo de $8 \%$ de sílica ativa e $0,7 \%$ de aditivo plastificante, sendo estas porcentagens em relação ao cimento.

Tabela 3 - Traço e misturas do CAD com e sem resíduo

\begin{tabular}{|l|c|}
\hline Mistura A (CAD “Padrão”) & \% \\
\hline Traço (em peso): 1: 2,5: 2,0 & - \\
\hline Agregado miúdo: Areia Grossa & 100 \\
\hline Agregado miúdo: Resíduo de Concreto & 0 \\
\hline Mistura B (CAD com 50\% de resíduo) & - \\
\hline Traço (em peso): 1: 2,5: 2,0 & - \\
\hline Agregado miúdo: Areia Grossa & 50 \\
\hline Agregado miúdo: Resíduo de Concreto & 50 \\
\hline Traço C (CAD com 100\% de resíduo) & - \\
\hline Traço (em peso): $1: 2,5: 2,0$ & - \\
\hline Agregado miúdo: Areia Grossa & 0 \\
\hline Agregado miúdo: Resíduo de Concreto & 100 \\
\hline
\end{tabular}

Anteriormente foram testados outros traços, porém devido à análise da aparência visual do concreto (HELENE e TERZIAN, 1992) estes foram mudados até que chegasse ao traço da Tabela 3. As dosagens estudadas foram obtidas de tal forma que a quantidade de cimento ficasse em torno de $500 \mathrm{~kg}$ por $\mathrm{m}^{3}$. Isto foi feito para 
diminuir as fissuras no concreto e ao mesmo tempo não apresentar um traço pobre, o que acarretaria em menor resistência, aumentando a exsudação, diminuindo a permeabilidade e a coesão.

$\mathrm{O}$ agregado graúdo constituiu de uma mistura efetuada com brita 01 e 00 . Para a determinação adequada da mistura entre as duas faixas granulométricas de pedras britadas utilizou-se o método da NBR NM 45:2006 - Agregado em Estado Compactado Seco - Determinação da Massa Unitária. (ABNT, 2006).

A determinação da massa unitária foi feita individualmente em cada mistura de agregados, contendo diferentes teores de cada fração das britas 00 e 01 (Tabela 4). Na primeira coluna da Tabela 4 tem-se a variação adotada (\%) na mistura dos dois agregados. Na segunda e terceira colunas estão apresentadas as quantidades de agregados necessárias para obter a proporção correspondente e ser suficiente para encher o recipiente, podendo haver sobra da mistura. A coluna de acréscimo da pedra britada $\mathrm{n}^{0} 00$ é correspondente à massa desse agregado, necessária para passar de uma composição (\%) para a seguinte, aproveitando sempre a mistura realizada anteriormente, ou seja, a contida dentro do recipiente e da sobra se for o caso. Na coluna de massa total do recipiente o valor da tara é de $0,37 \mathrm{~kg}$ e para o cálculo da massa unitária no estado compactado o volume do recipiente é de $8,8 \mathrm{dm}^{3}$.

A escolha da composição considerada "ideal" baseia-se no maior valor obtido para a massa unitária no estado compactado, calculada conforme Equação 1.

Tabela 4 - Determinação da composição "ideal” entre pedra britada 00 e 01, através da massa unitária no estado compactado seco

\begin{tabular}{|c|c|c|c|c|c|}
\hline $\begin{array}{c}\text { Composição } \\
\text { entre } \\
\text { agregados } \\
(01 \mathrm{e} 00) \\
(\%)\end{array}$ & $\begin{array}{c}\text { Quantidade de } \\
\text { pedra britada } \\
01\end{array}$ & $\begin{array}{c}\text { Quantidade de } \\
\text { pedra britada } \\
(\mathrm{kg})\end{array}$ & $\begin{array}{c}\text { Acréscimo de } \\
\text { pedra britada } \\
00 \text { para obter } \\
\text { a composição } \\
\text { desejada } \\
(\mathrm{kg})\end{array}$ & $\begin{array}{c}\text { Massa total } \\
\text { do recipiente } \\
\text { (agregado }+ \\
\text { tara) }(\mathrm{kg})\end{array}$ & $\begin{array}{c}\text { Massa } \\
\text { unitária no } \\
\text { estado } \\
\text { compactado } \\
(\text { Equação 1) } \\
\left(\mathrm{kg} / \mathrm{dm}^{3}\right)\end{array}$ \\
\hline $100 / 0$ & 12,15 & 0 & 1,35 & 12,52 & \\
\hline $90 / 10$ & 12,15 & 1,35 & 1,27 & 1,47 \\
\hline $80 / 20$ & 12,15 & 3,04 & 1,69 & 14,09 & 1,56 \\
\hline $70 / 30$ & 12,15 & 5,21 & 2,17 & 14,37 & 1,59 \\
\hline $60 / 40$ & 12,15 & 8,10 & 2,89 & 14,21 & 1,57 \\
\hline
\end{tabular}

massa unitária no estado compactado $=\frac{\text { massa total } \text { do } \text { recipiente }- \text { tara }}{\text { volume } \text { do } \text { recipiente }}\left(\mathrm{kg} / \mathrm{dm}^{3}\right)$

A quantidade de água para cada traço foi determinada fixando-se o abatimento dos concretos, no ensaio do cone, em $100 \pm 10 \mathrm{~mm}$, tanto para mistura com resíduo quanto para mistura sem resíduo. Isso foi feito para verificar a influência do resíduo na relação água/cimento $(\mathrm{a} / \mathrm{c})$. 


\subsection{Ensaio do Cone}

O abatimento do concreto fresco foi medido usando o equipamento padrão do ensaio do cone. Para cada traço se estimou uma relação água/cimento e de acordo com a medida do abatimento diminuiu ou aumentou essa relação, até chegar ao abatimento desejado.

\subsection{Resistência à Compressão}

Os ensaios de resistência à compressão axial foram realizados aos 37,28 e 60 dias, seguindo-se as prescrições da NBR5739/1994. (ABNT, 1994). Os corpos-deprova foram confeccionados em molde cilíndrico e tiveram as dimensões $10 \mathrm{~cm}$ de diâmetro por $20 \mathrm{~cm}$ de comprimento. Os cilindros foram removidos do molde após 24 horas e levados à câmara úmida até a hora do ensaio. Todos os corpos de prova da mesma mistura foram confeccionados em um mesmo lote para todas as idades, a fim de diminuir dispersão dos resultados.

\subsection{Resistência à Flexão}

Os ensaios de resistência à flexão foram realizados aos 3, 7, 28 e 60 dias, (ensaio dos dois cutelos), segundo a NBR 12142/1991 (ABNT, 1991)

Os corpos de prova, para resistência à flexão, foram confeccionados conforme a NBR 5738/1993 (ABNT, 1993). Estes tiveram a forma prismática e as dimensões de $150 \times 150 \times 500 \mathrm{~mm}^{3}$. Os prismas foram removidos do molde após 24 horas e levados à câmara úmida até a hora do ensaio. Todos os corpos de prova da mesma mistura foram confeccionados em um mesmo lote para todas as idades, a fim de diminuir dispersão dos resultados.

\section{RESULTADOS E DISCUSSÃO}

A composição "ideal" para a mistura de brita 00 e brita 01 foi de 30 e $70 \%$, respectivamente (Tabela 4). Essa foi à porcentagem que apresentou maior massa unitária no estado compactado.

Para as diferentes idades, misturas e ensaios foram confeccionados quatro corpos de prova para cada caso. Na Figura 1 são apresentados os resultados de resistência à compressão, obtidos nos ensaios realizados às idades de 03, 07, 28 e 60 dias, para o CAD sem resíduo, para o CAD com $50 \%$ de resíduo e para o CAD com substituição total da areia por resíduo de concreto (100\% resíduo). Na Tabela 5 se observam os valores mostrados na Figura 1 para o ensaio de compressão e também os resultados do ensaio de flexão. 


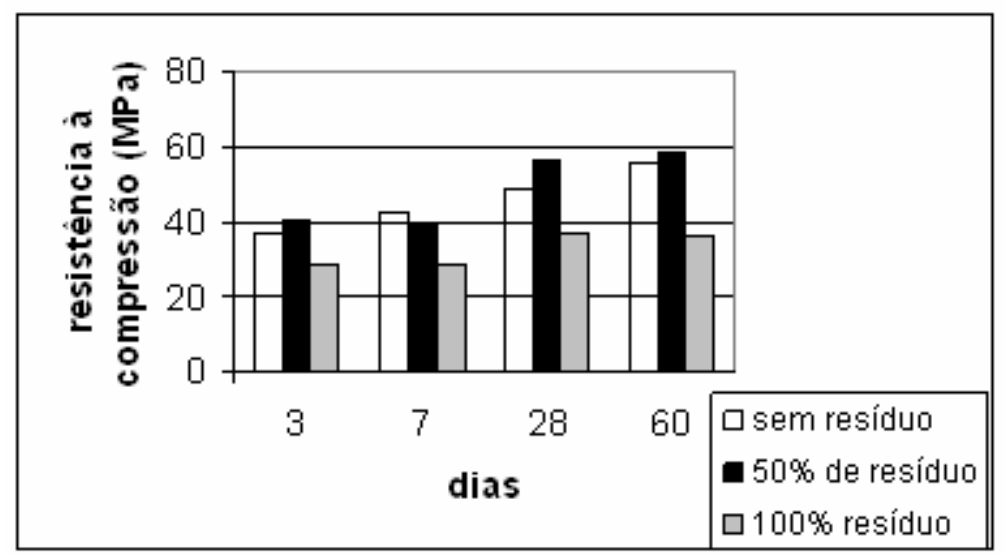

Figura1. Resultados de resistência á compressão aos 03, 07, 28 e 60 dias

Tabela 5 - Valores de Resistência à compressão e à tração na flexão para o CAD sem resíduo, com $50 \%$ de resíduo e com substituição total da areia por resíduo de concreto.

\begin{tabular}{|c|c|c|c|c|c|c|c|c|c|}
\hline \multirow{3}{*}{$\begin{array}{l}\text { Tipo de } \\
\text { mistura }\end{array}$} & \multirow{3}{*}{$\begin{array}{l}\text { Tipo } \\
\text { de } \\
\text { valor }\end{array}$} & \multirow{2}{*}{\multicolumn{4}{|c|}{$\begin{array}{l}\text { Resistência à compressão (MPa) } \\
\text { Idade (dias) }\end{array}$}} & \multicolumn{4}{|c|}{ Resistência à tração $(\mathrm{MPa})$} \\
\hline & & & & & & \multicolumn{4}{|c|}{ Idade (dias) } \\
\hline & & 03 & 07 & 28 & 60 & 03 & 07 & 28 & 60 \\
\hline \multirow{4}{*}{$\begin{array}{l}\text { sem } \\
\text { resíduo }\end{array}$} & $\mathrm{VM}^{1}$ & 36,92 & 42,49 & 49,18 & 56 & 6,18 & 6,62 & 8,49 & 8,64 \\
\hline & $\mathrm{DP}^{2}$ & 03,59 & 14,08 & 09,73 & 10 & 0,47 & 0,20 & 1,02 & 0,32 \\
\hline & $\mathrm{VMa}^{3}$ & 40,57 & 56,58 & 58,91 & 66 & 6,65 & 6,83 & 9,51 & 8,96 \\
\hline & $\mathrm{VMi}^{4}$ & 33,33 & 28,41 & 39,45 & 45 & 5,70 & 6,42 & 7,47 & 8,33 \\
\hline \multirow{4}{*}{$\begin{array}{l}50 \% \\
\text { resíduo }\end{array}$} & VM & 40,57 & 40,32 & 56,77 & 58 & 6,44 & 6,98 & 8,09 & 7,47 \\
\hline & DP & 11,46 & 16,64 & 16,87 & 9 & 0,08 & 0,31 & 0,66 & 0,81 \\
\hline & $\mathrm{VMa}$ & 52,03 & 56,96 & 73,64 & 67 & 6,52 & 7,29 & 8,75 & 8,28 \\
\hline & VMi & 29,11 & 23,68 & 39,89 & 48 & 6,37 & 6,67 & 7,43 & 6,66 \\
\hline \multirow{4}{*}{$\begin{array}{l}100 \% \\
\text { resíduo }\end{array}$} & VM & 29,28 & 29,11 & 37,05 & 36 & 4,56 & 4,93 & 5,85 & 5,85 \\
\hline & DP & 1,54 & 1,64 & 1,82 & 2 & 0,50 & 0,54 & 0,53 & 0,71 \\
\hline & Vma & 30,82 & 30,75 & 38,87 & 38 & 5,06 & 5,47 & 6,38 & 6,56 \\
\hline & VMi & 27,74 & 27,48 & 35,23 & 34 & 4,06 & 4,40 & 5,32 & 5,14 \\
\hline
\end{tabular}

Observa-se que aos 3 dias a máxima resistência média ocorreu para o ensaio realizado nos corpos-de-prova confeccionados com $50 \%$ de resíduo, entretanto houve um grande desvio padrão para os resultados conforme se pode observar na Tabela 5.

Devido a este desvio o valor mínimo obtido, em relação aos corpos de prova sem resíduo e com $50 \%$ de resíduo foi para os corpos de prova com $50 \%$ de resíduo. Aos 7 dias este resultado inverteu e a máxima resistência média ocorreu para o ensaio realizado nos corpos-de-prova confeccionados sem resíduo, entretanto, novamente devido ao desvio, o valor máximo foi para o corpo de prova com $50 \%$ de resíduo. Aos 28 dias o valor da máxima resistência média foi para o CAD com $50 \%$ de 
resíduo e novamente a dispersão foi alta, resultando que o menor valor entre o CAD sem e com 50\% de resíduo foi muito próximo, o mesmo ocorreu aos 60 dias. Portanto, ao se analisar o desvio padrão, se pode afirmar que os intervalos de valores para esses corpos de prova são o mesmo.

Quando se compara os resultados com os corpos de prova confeccionados com $100 \%$ de resíduo, estes apresentaram menor resistência para todas as idades. A elevada dispersão dos resultados deve estar ocorrendo devido à microestrutura do material, que apresenta grãos grandes e, quanto maior o grão, maior deverá ser a dispersão (SAKAI, 1987; LEMAISTRE, 1998). Essa mesma dispersão não está ocorrendo nos corpos de prova com $100 \%$ de resíduo, isto deve estar acontecendo porque a energia para a ruptura destes materiais é bem menor, o que faz com que o defeito do material não seja tão significativo, pois a própria estrutura já se rompe com baixas tensões. O mesmo ocorreu para a resistência à flexão conforme será mostrado na seqüência, ou seja, a dispersão dos resultados foi bem menor que a de resistência à compressão, pois a resistência à flexão apresenta carga de rupturas com valores bem mais baixos.

Na Figura 2 são apresentados os resultados de resistência à flexão, obtidos dos ensaios realizados às idades de 03, 07, 28 e 60 dias, para o CAD sem resíduo, para o CAD com substituição de $50 \%$ do agregado miúdo por resido de concreto e para o CAD com $100 \%$ de substituição da areia por resíduo de concreto. Observa-se que aos 03 e 07 dias a máxima resistência média ocorreu para o ensaio realizado nos corposde-prova confeccionados com areia e resíduo de concreto.

Aos 28 e 60 dias o CAD que continha somente areia apresentou resistência média maior que o CAD que continha areia e resíduo. Quando se analisaram os valores do desvio padrão (Tabela 6), observou-se que aos três dias o valor máximo ocorreu para o corpo de prova sem resíduo e aos 7 dias o valor mínimo ocorreu para o corpo de prova com $50 \%$ de areia. Aos 28 dias o valor mínimo foi praticamente o mesmo para os traços com $50 \%$ de resíduo e sem resíduo. Pode-se afirmar que os valores estão dentro da mesma faixa. Somente os resultados com os corpos de prova confeccionados com $100 \%$ de resíduo apresentaram menor resistência para todas as idades como ocorreu com a resistência à compressão.

Com base nos resultados deste estudo se pode dizer que a substituição de $50 \%$ da areia natural por concreto reciclado não influenciou os resultados de resistência. Esse resultado está de acordo com Poon et al. (2002, 2004), que fizeram o ensaio e substituíram o agregado natural por 20,50 e $100 \%$ de resíduo de construção no concreto normal para os agregados secos ao ar, como o deste estudo.

$\mathrm{Tu}$ et al. (2006) mediram a resistência para o CAD com reciclado e obtiveram queda de resistência. Neste estudo, entretanto, o agregado natural foi substituído integralmente pelo reciclado.

Ajdukiewicz e Kliszecewicz (2002) também fizeram um estudo com substituição do agregado natural pelo agregado reciclado de concreto para o CAD e concluíram que a resistência a tração teve uma queda com o agregado reciclado, 
entretanto, novamente a substituição foi total tanto para areia quanto para o agregado graúdo. Nos resultados aqui obtidos concluiu-se que as propriedades do concreto usado como agregado reciclado teve influência significante nas propriedades mecânicas do CAD com resíduo, podendo, dependendo de o reciclado, alcançar uma resistência à compressão mais alta que para o CAD sem resíduo.

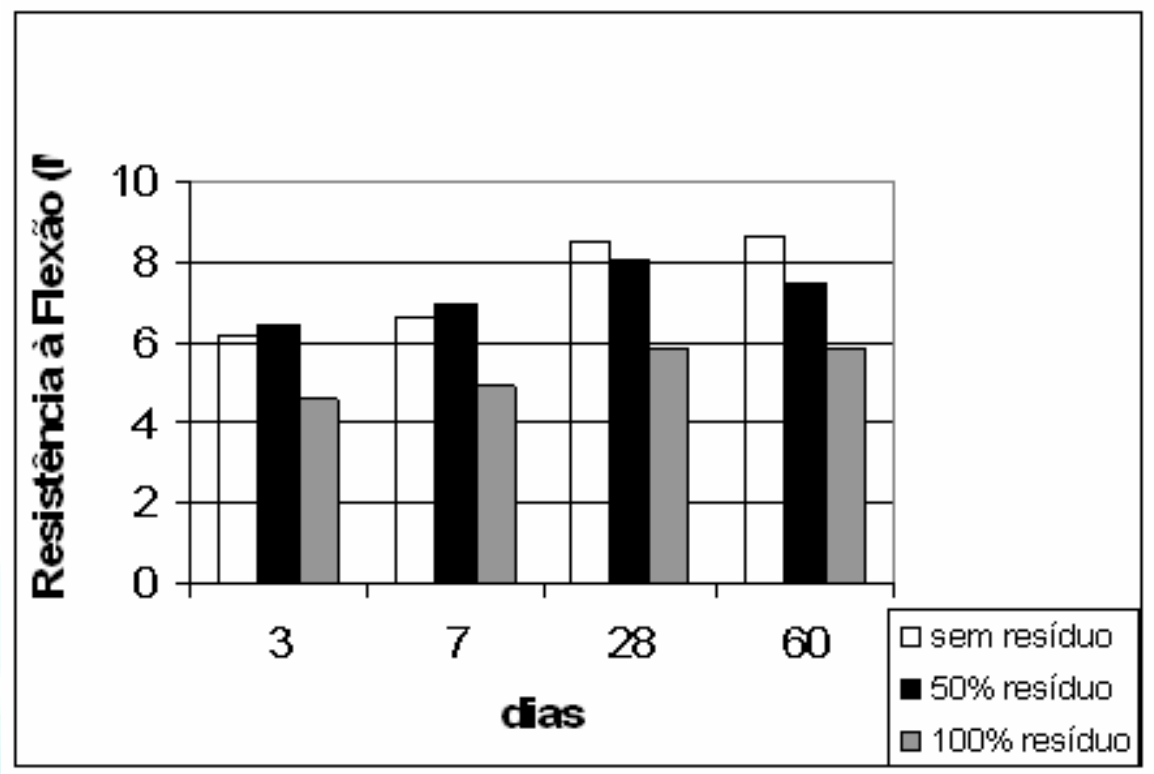

Figura 2. Resultados de resistência á flexão aos 03, 07, 28, 60 dias

$\mathrm{Na}$ Figura 3 apresentam-se os valores da relação a/c, para os concretos confeccionados. Verifica-se que quase não houve diferença na relação a/c entre o CAD com resíduo e o sem resíduo, sendo que no CAD sem resíduo a relação a/c foi de 0,31 e no $\mathrm{CAD}$ com resíduo foi de 0,33 e o CAD com $100 \%$ de resíduo não apresentou variação em relação ao CAD com $50 \%$ de resíduo.

Este resultado está de acordo com Adjukiewicz e Kliszecewicz (2002), os quais concluíram que as mudanças no conteúdo água/cimento podem ser relativamente pequenas para o CAD quando se usou o resíduo de concreto como agregado.

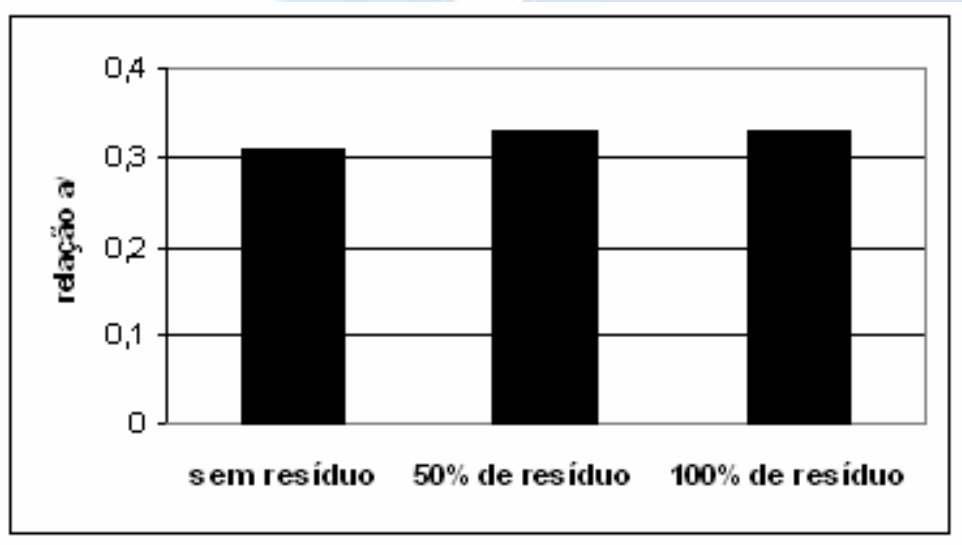

Figura 3. Relação a/c para o CAD sem resíduo e com resíduo 


\section{CONCLUSÕES}

Em vista dos resultados obtidos e das discussões apresentadas, conclui-se que a incorporação do resíduo substituindo $50 \%$ de areia exerceu uma alteração bastante pequena na relação água/ cimento das composições estudadas, havendo necessidade de uma maior quantidade de água para se obter um abatimento igual ao obtido para o concreto sem adição de resíduo. Porém não houve diminuição de resistência; para o CAD com $50 \%$ de resíduo. Este obteve praticamente a mesma resistência à compressão e à flexão, sendo que o mesmo não ocorreu para o CAD com $100 \%$ de resíduo. Este resultado está de acordo com outros estudos citados na literatura para o concreto normal.

Em relação à resistência à tração e à flexão, concluiu-se que os resíduos de concreto podem ser utilizados, de maneira satisfatória, em substituição parcial à areia natural, na confecção do concreto de alto desempenho. Esta substituição pode configurar-se numa excelente alternativa, plenamente inserida no contexto do desenvolvimento sustentável, para o aproveitamento deste resíduo.

Com os dados obtidos neste estudo, verificou-se que existe possibilidade técnica para o aproveitamento dos resíduos de concreto na composição do concreto de alto desempenho. Torna-se necessário, contudo, avançar no estudo de outras propriedades, comportamento e aplicações do CAD com resíduos de construção para uma aplicação mais segura e em escala maior.

\section{AGRADECIMENTOS}

- À FUNDUNESP, Silmix da Camargo Corrêa Cimentos S.A., Holcim e CESP.

\section{REFERÊNCIAS}

ABNT - ASSOCIAÇÃO BRASILEIRA DE NORMAS TÉCNICAS. Cimento Portland de alta resistência inicial NBR 5733. Rio de Janeiro, 5p, 1991.

ABNT - ASSOCIAÇÃO BRASILEIRA DE NORMAS TÉCNICAS. Concreto Determinação da resistência à tração na flexão em corpos-de-prova prismáticos. NBR 12142. Rio de Janeiro, 3p, 1991.

ABNT - ASSOCIAÇÃO BRASILEIRA DE NORMAS TÉCNICAS. Moldagem e cura de corpos-de-prova cilíndricos ou prismáticos de concreto. NBR 5738, Rio de Janeiro, 9p, 1993. 
ABNT - ASSOCIAÇÃO BRASILEIRA DE NORMAS TÉCNICAS. Concreto Ensaio de compressão de corpos-de-prova cilíndricos. NBR 5739. Rio de Janeiro, 4p, 1994.

ABNT - ASSOCIAÇÃO BRASILEIRA DE NORMAS TÉCNICAS. Agregados Determinação da massa unitária e do volume de vazios. NBR 45, Rio de Janeiro, 8p, 2006.

AJDUKIEWICZ, A.; KLISZCZEWICZ, A. Influence of recycled aggregates on mechanical properties of HS/HPC. Cement and Concrete Composites, v.24. p.269279, 2002.

ATIS, C. D. Strength properties of high-volume fly ash roller compacted and workable concrete and influence of curing condition. Cement and Concrete Research, v.36, n.6, p.1112-1121, 2005.

BRITO, J.; PEREIRA, A. S.; CORREIA, J. R. Mechanical behaviour of nonstructural concrete made with recycled ceramic aggregates. Cement and Concrete Composites, v.27, n.4, p.429-433, 2005.

CHANDRA, S. (Ed.) - Waste materials used in concrete manufacturing. Noyes Publications, New Jersey, USA, 1997.

HELENE, P. R. L.; TERZIAN, P. Manual de Dosagem e Controle de Concreto. Editora PINI, Brasília DF: SENAI, 349 p, 1992.

HUANG, B.; SHU, X.; LI, G. Laboratory investigation of portland cement concrete containig recycles asphalt pavements. Cement and Concrete Research, v.35, n.10, p.2008-2013, 2005.

LEMAISTRE, H. Étude des propriétés thermomécaniques de divers réfractaires. Thèse.1998. (Doctorat en Génie des Matériaux) - École Doctorale Matériaux, Institut National de Sciences Appliquées, Lyon, 1998.

POON, C. S.; SHUI, Z. H.; LAM, L. Strength of concretes prepared with natural and recycled aggregates at different moisture condition. In: PROCEEDINGS... Of the International Conference on Advances in Building Technology 4-6 december 2002, Hong Kong, China, p. 1407-1414, 2002.

POON, C. S.; SHUI, Z. H.; LAM.; KOK, H., KOU S.C. Influence of moisture states of natural and recycled aggregates on slump and compressive strength of concrete.

Cement and Concrete Research, v.34, p.31-36, 2004. 
SAKAI, M. Fracture Mechanics of Refractory Materials. Taikabutsu Overseas, v.8, n.2, p. 4-12, 1987.

SCOARIS, M. R. Cura Térmica dos Concretos com Fibras: Avaliação do

Comportamento Mecânico e Estrutural. Relatório de Exame Geral de Qualificação apresentado à Faculdade de Engenharia de Ilha Solteira - UNESP, 2004.

TU, T. Y.; CHEN Y. Y.; HWANG C. L. Properties of HPC with recycled aggregates. Cement and Concrete Research, v.36, p.943-950, 2006. 